Optimising the hydrophobicity of sands by silanisation and powder coating

\begin{abstract}
Sands are naturally hydrophilic granular materials, yet, rendering them hydrophobic could lend them to a wide range of geotechnical applications. This study describes a powder coating procedure performed after chemically modifying the surfaces of coarse, medium and fine sands and examines its effect on their hydrophobicity. The purpose is to render these granular materials more hydrophobic than what is conventionally achieved by chemical methods using a simple technique. The procedure consists of first silanising both the sands and silica powder at a similar concentration by means of an organosilane to modify their surface chemistry, then the silica powder is adhered to the sands at a mass mixing ratio to alter their hydrophobicity. Irrespective of the concentrations and mixing ratios, the powder coating procedure enhances the hydrophobicity of sands in comparison to the sole use of the chemical method. Changes in the morphology of the sand grains, such as their particle size, particle shape and surface roughness resulting from the powder coating procedure are examined by means of dynamic image analysis, profilometry and scanning electron microscopy. The effects of surface chemistry, surface roughness and air on the hydrophobicity of the sands are discussed based on theoretical wetting models to analyse the experimental results.
\end{abstract}

\title{
Keywords
}

Chemical properties; laboratory tests; particle-scale behavior; sands; ground improvement 


\section{List of notations}

$\begin{array}{ll}\text { CA } & \text { contact angle } \\ \gamma_{\mathrm{wa}} & \text { interfacial force between the water-air } \\ \gamma_{\mathrm{sw}} & \text { interfacial force between the solid-water } \\ \gamma_{\mathrm{sa}} & \text { interfacial force between the solid-air } \\ \theta_{\mathrm{y}} & \text { Young's contact angle } \\ \theta_{\mathrm{w}} & \text { Wenzel's contact angle } \\ r & \text { roughness factor } \\ \theta_{\mathrm{cb}} & \text { Cassie-Baxter's contact angle } \\ f_{1} & \text { area fraction of the solid in contact with the water drop } \\ k^{-1} & \text { capillary length of water } \\ \rho & \text { density of water } \\ g & \text { gravitational constant } \\ H & \text { water entry pressure } \\ r_{\mathrm{c}} & \text { capillary radius } \\ \text { DMDCS } & \text { dimethyldichlorosilane } \\ \text { PDMS } & \text { polydimethylsiloxane } \\ \text { DIA } & \text { dynamic image analyser } \\ D_{50} & \text { median value of cumulative distribution for particle size } \\ S_{50} & \text { median value of cumulative distribution for sphericity } \\ A r_{50} & \text { median value of cumulative distribution for aspect ratio } \\ C V_{50} & \text { median value of cumulative distribution for convexity } \\ \text { SEM } & \text { scanning electron microscope } \\ R_{a} & \text { surface roughness } \\ C & \text { critical concentration of the silica powder }\end{array}$




\section{Introduction}

3 Rendering soil particles hydrophobic by mixing with chemical additives is known to reduce the expansion of swelling clays (Hernandez et al. 2005), influence the capillary rise in fine silt

5 (Orozco and Caicedo, 2017) and in sands, to alter their electrical conductivity (Dong and

6 Pamukcu, 2015) and their evaporation rate (Kim et al. 2015). With sands, hydrophobisation can

7 also lead to several geotechnical applications, as barriers at the soil-atmosphere-vegetation and at soil-structure interfaces. For example, their potential use in solid waste landfills as covers to prevent water infiltration has been proposed by Subedi et al. (2012). The efficiency of using hydrophobic sands for such a system will depend on the water entry pressure i.e. the critical pressure at which water displaces air, which is a function of the sand properties such as porosity (Lee et al. 2015) and is positively correlated to the extent of hydrophobicity (Carillo et al. 1999). The water entry pressure, $H$ is related to the contact angle (CA) according to equation

14 (1) where $\gamma_{w a}$ is the interfacial force between the water-air phases, $r_{c}$ is the capillary radius, $g$ is the gravitational constant and $\rho$, the density of water. Furthermore, hydrophobicity in sands also influences their water retention properties (Keatts et al. 2018). Key advantages for using hydrophobic sands are as follows: 1) High gas permeability while remaining impermeable to liquids. 2) Volumetric stability, unlike clays that are prone to swelling and shrinking (including desiccation cracks). 3) Reusing waste-derived materials such as glass for hydrophobisation.

Hydrophobic materials, characterised by a CA $>90^{\circ}$ have found applications in numerous fields as microfluidics devices, self-cleaning surfaces and textile fabrics (Grunze, 1999; Blossey, 2003; Zimmermann et al. 2008). On a flat surface, hydrophobicity is exclusively a function of the surface chemistry, with surfaces treated with fluoropolymers being the most hydrophobic

27 (Lafuma and Quéré, 2003; Darmanin and Guittard, 2015); the CAs reported on these surfaces do not exceed $120^{\circ}$. On rough surfaces, the surface texture has been shown to contribute to hydrophobicity (Wenzel, 1936; Lafuma and Quéré, 2003). A commonly cited example throughout the literature is the lotus leaf, displaying multi-scales of roughness, conducive to a 
31 high CA of $150^{\circ}-160^{\circ}$ (Feng et al. 2002; Darmanin and Guittard, 2015). Numerous experimental 32 studies have been devoted to functionalising materials using both chemical and physical 33 methods such as altering their surfaces by microscale (e.g. using silicon pillars) and nanoscale 34 (e.g. using carbon nanotubes) modifiers to achieve high CAs, comparable to the lotus leaf 35 (Shirtcliffe et al. 2004; Wang et al. 2007; Zhang et al. 2015). However, adapting such physical 36 modifications to granular materials such as sands is not feasible due to the costs involved. 37 Therefore, for applications to geotechnical problems, user-friendly approaches that take into 38 account economic factors are needed. A new method is introduced in this paper to optimise the 39 hydrophobicity of sands through comparatively simple techniques. A brief overview of the 40 methods currently used to render granular soils hydrophobic is first given below.

Chemical methods to render sands hydrophobic include the use of agents such as fatty acids 43 (Subedi et al. 2012), waxes (Bardet et al. 2014), oils (Zhang et al. 2016) and organosilanes (Ng 44 and Lourenço, 2016). From a sample preparation perspective, mixing organosilanes in liquid 45 form with sands, being a single step process is the most straightforward and also a well46 documented method that has been shown to induce hydrophobicity in sands while also retaining 47 their non-biodegradability in water (Bachmann and McHale, 2009). Organosilanes can be 48 broadly classified as mono-functional (e.g. trimethylchlorosilane) and multi-functional (e.g. 49 dimethyldichlorosilane and octadecyltrichlorosilane). Mono-functional organosilanes have a 50 single reactive site which can react with only one hydroxyl group $(-\mathrm{OH})$ on the sands whereas 51 the reaction between multi-functional organosilanes and sands lead to complex molecular 52 configurations as a result of both vertical and horizontal polymerisations (Tripp and Hair, 1991). 53 A comparison between different organosilanes used to hydrophobise sands was investigated by 54 Chan and Lourenço (2016). They concluded that the use of multi-functional organosilanes such 55 as dimethyldichlorosilane (DMDCS) required lesser amount of chemical and thus was more cost 56 effective to achieve the maximum CA. The synthesis of hydrophobic sands using organosilanes 57 is known as silanisation. With DMDCS, the basic mechanism first involves reaction with water 58 (present in the atmosphere and/or on the sands) to give polydimethylsiloxane (PDMS) which is 59 a soft polymeric coating (Liu et al. 2019) and hydrogen chloride according to equation (2) where $60 n$ is the number of DMDCS repeating units. The hydroxyl groups on the sands then react with 

al., 2007).

63

$$
n\left[\left(\mathrm{CH}_{3}\right)_{2} \mathrm{SiCl}_{2}\right]+n\left[\mathrm{H}_{2} \mathrm{O}\right] \rightarrow\left[\left(\mathrm{CH}_{3}\right)_{2} \mathrm{O}\right]_{n}+2 n \mathrm{HCl}
$$

66 Physical methods to optimise the hydrophobicity of sands are designed based on particle 67 characteristics such as particle size, particle shape and surface roughness. They are typically not used on their own, but combined with chemical treatment of the soil grains. The dependency of the hydrophobicity of soils on particle size has been shown in several studies such as Bachmann et al. (2003) with the finer fractions exhibiting a larger CA than the coarser ones while Saulick et al. (2018) showed that for a given surface chemistry of granular materials comprising of sands and artificial particles synthesised with DMDCS, angular particles were more hydrophobic. With granular materials, two scales of surface roughness can be defined, namely the surface roughness of single particles as investigated by Yang et al. (2016) and the surface roughness of a series of particles, quantified by Saulick et al. (2018) as a function of both particle size and particle shape. The latter have shown that for a given surface chemistry of granular materials, the finer and more angular-shaped particles translate into a smaller surface roughness parameter and are more hydrophobic.

The research in this paper presents a new method for optimising or controlling the hydrophobicity of sands by combining chemical treatment by means of an organosilane (chemical method) and surface alteration by powder coating (physical method) in order to render them more hydrophobic than what is conventionally achieved by chemical methods.

84 Techniques comprising dynamic image analysis, scanning electron microscopy and optical interferometry were used to characterise the morphology of the grains before and after powder coating. Although the methods used, individually or combined are known to influence considerably the CAs of sands, the combined effect of chemically and physically modifying their surfaces on hydrophobicity has not been investigated to date. To achieve this aim, sands of different grading were first chemically modified at different concentrations and then physically 
were only chemically modified and any differences in hydrophobicity attributed to the powders coated on the sands. The results are then compared with theoretical wetting models used in physical chemistry such as the Wenzel and Cassie-Baxter models which include effects of surface chemistry, surface roughness and air on the hydrophobicity of granular materials, thus giving an insight into the micro-mechanisms underlying sand wettability.

\section{Theoretical background}

98

Three main theoretical models exist which describe the wettability of surfaces; they are the Young's model that assumes the surface to be flat, Wenzel's and Cassie-Baxter's models that consider the roughness of the surfaces.

102

\subsection{Wettability of flat surfaces: Young's model}

104

A drop of water deposited on a solid in air will either spread or adopt a spherical cap-like shape depending on the three interfacial forces on it. They are $\gamma_{w a}, \gamma_{s w}$ and $\gamma_{s a}$ corresponding respectively to the interfacial forces between the water-air, solid-water and solid-air phases. At the three-phase point, resolving the interfacial forces horizontally assuming mechanical equilibrium generates a relationship with the CA on the wet side of the water drop, which is a measure of the wettability of the solid (Figure 1a).

110

111 For a drop of water on an ideal (smooth, homogenous, inert, insoluble, non-porous and non-

112 deformable) flat solid, a relationship between the interfacial forces and an angle, $\theta_{y}$ can be 113 established where $\theta_{\mathrm{y}}$ is defined as Young's contact angle (Young, 1805):

114

\section{$117 \quad 2.2$ Wettability of rough surfaces: Wenzel's model}

118 The model developed by Wenzel (1936) takes into account the effect of surface roughness and

119 modifies Young's equation by a material-independent roughness factor, $r$ to give Wenzel's contact angle, $\theta_{\mathrm{w}}$ according to the following equation: 
124 The quantity $r$ in equation (4) is defined as the ratio of actual (including the surface protrusions) 125 and projected areas of the solid. The Wenzel model assumes a complete wetting where a drop 126 of water completely fills the grooves (Figure 1b). Because the value of $r$ always exceeds unity, 127 equation (4) implies that for a solid with $\theta_{y}$ less (greater) than the $90^{\circ}$ threshold, hydrophobicity 128 will subdue (intensify).

129

\subsection{Wettability of rough surfaces: Cassie-Baxter's model}

131 Cassie-Baxter's model considers a wetting regime where a drop of water deposited on a solid does not fill the grooves completely and has air trapped between the solid and water interface

133 (Figure 1c) which leads to an enhancement in hydrophobicity. This is due to the synergistic decrease in the area fraction of the solid in contact with the water drop $\left(f_{1}\right)$ and increase in the area fraction of air in contact with the water drop $\left(1-f_{1}\right)$. To account for this difference in chemistry, Cassie and Baxter (1944) expressed the contact angle, $\theta_{c b}$ as follows:

140 These three models can be used to compare with the contact angles measured in the laboratory

141 to assess their suitability to describe the hydrophobicity of granular materials, while also giving 142 some insight into what controls the wettability of soils. The following section describes the experimental programme, which consisted of changing the surface properties of a quartz sand

144 by chemical treatment (silanisation) and surface alteration (by powder coating), and quantifying 145 the changes in terms of contact angle and surface morphology.

\section{3. Experiments}

\section{$148 \quad 3.1$ Tested materials}

149 Fujian sand, a commercially available sand with a high proportion of silica was used. Silica 150 powder was chosen to coat the sands due to its widespread use in the construction industry for 
151 producing functional cementitious mixtures (Bentz et al., 2017). Both the sands and the silica 152 powder were sourced from a quarry located at Xiamen, Fujian, China. After a dry sieve analysis, 153 three sand fractions were isolated based on their particle sizes: coarse sand (600-1180 $\mu \mathrm{m})$, 154 medium sand $(212-300 \mu \mathrm{m})$ and fine sand $(63-212 \mu \mathrm{m})$. The median particle size $\left(D_{50}\right)$ of the 155 silica powder was $23 \mu \mathrm{m}$. The sands and silica powder were initially washed and oven-dried at $15680^{\circ} \mathrm{C}$ prior to any tests. Microscope slides made of soda lime-silica glass (with comparable 157 chemistry to the sands and silica powder), and of dimensions $76 \times 26 \mathrm{~mm}$ by $1 \mathrm{~mm}$ thick, were 158 used as references for ideal flat solids.

159

160

\subsection{Silanisation}

161 A liquid-phase silanisation using DMDCS (Acros Organics, Morris Plains, NJ, USA) was carried out to hydrophobise the sands, silica powder and microscope slides. The silanisation reactions were carried out in a fume cupboard at 14 concentrations (defined as the mass ratio of DMDCS added to the sands or the silica powder expressed as a percentage) to identify the critical concentration-the smallest concentration of DMDCS needed to reach the maximum CA. The concentrations used were between $0.00265 \%$ and $3.71 \%$. To render the microscope slides hydrophobic, a total of $20 \mu$ l of DMDCS was dispensed from a single channel pipette (Pipetman P100 from Gilson®, Villiers-le-Bel, France) and left to react for 24 hours.

\subsection{Powder coating procedure}

171 The first step in the powder coating procedure involved isolating the effect of surface chemistry

172 of the sands and the silica powder by carrying out the silanisation at the critical concentration of 173 the silica powder $(C)$. Afterwards, the silanised sand and silanised silica powder were mixed at a 174 mass ratio and the excess silanised silica powder discarded. Next, the mixture was washed on 175 a $63 \mu \mathrm{m}$ mesh. To accelerate the evaporation process and obtain the powder-coated sands, the 176 mixture was oven-dried at $80^{\circ} \mathrm{C}$ for 16 hours, to avoid thermal degradation of the PDMS coating 177 (Camino et al., 2001). The powder-coated sands are a result of the adhesion between the 178 silanised sand and the silanised silica powder. This involves molecular bonding consisting of 179 intermolecular bonds such as Van der Waal forces when the interfaces are brought in contact 180 supplemented by covalent bonds formed at the interfaces as a result of vertical and horizontal 

coupling have also been reported (e.g. in Brown (2000)) to contribute to adhesion in polymers.

183 A schematic illustration of the powder coating procedure is shown in Figure 2.

185 Two parameters were investigated when carrying out the powder coating procedure: (i) the concentration at which the sands and the silica powder was silanised. This concentration was increased from $C$ to $2 C$ and $7 C$, the critical concentration $C$ being considered the minimum required to achieve consistent hydrophobicity. (ii) the mass ratio at which the silanised sand and silica powder was mixed. The ratio of silanised sand and silica powder was first set as 1 to 1 and then raised to 1 to 3 . A comparison of the CAs obtained with the values predicted with the models including or not surface roughness will then give some insight into the mechanics of soil wettability at the grain scale.

\subsection{Contact angle measurement}

195 A goniometer (Drop Shape Analyzer 25, KRÜSS GmbH, Hamburg, Germany) was used to measure the CAs of the materials via the sessile drop method, a method widely used in the soil and material sciences. Sample preparation for each of the granular materials was carried out according to the technique proposed by Bachmann et al. (2000) by fixing a monolayer of the granular materials on a microscope slide with double-sided tape attached to it. Ten micro-liters $(10 \mu \mathrm{L})$ drops of deionised water were placed on each sample using the automatic dispenser of the goniometer and images were obtained from a charged coupled device camera positioned laterally to the samples. To restrict the influence of the shape of the sessile drop on CAs, the gaps between the granular materials should be less than $k^{-1}$, the capillary length of water $(2.7$ $\mathrm{mm})$. The capillary length of water is a characteristic length which depends on the interfacial force between the water-air phases $\left(\gamma_{\text {wa }}\right)$, density of water $(\rho)$ and the gravitational constant $(g)$ according to equation (6). Because the gaps between the granular materials in this study are 207 less than the capillary length of water, the influence of gravity on the drop shape can be 208 neglected. The tests were carried out in air at a temperature of $24^{\circ} \mathrm{C}$ and relative humidity of $20965 \%$. Although the sessile drop is a commonly used technique to evaluate CAs, their 210 determination retains an element of subjectivity linked for example to the positioning of the 
211 baseline. The semi-automated technique developed by Saulick et al. (2017) was applied to

212 evaluate the CAs using ImageJ, an open source image processing software. The mean value of

213 the ten measurements and the corresponding standard deviation on each sample were adopted

214 as the measured data.

215

216

$k^{-1}=\sqrt{\frac{\gamma_{w a}}{\rho g}}$

217

218

\subsection{Characterisation of particle size, shape and surface roughness}

219 The coating of sand grains by DMDCS and powder may alter their size and morphology. These

220 were examined before and after treatment by means of a dynamic image analyser and an

221 interferometer for a quantitative description of size, shape and texture, and by scanning electron

222 microscopy for a qualitative assessment.

223

224 A dynamic image analyser (DIA), QICPIC ${ }^{\mathrm{TM}}$ (Sympatec GmbH, Clausthal-Zellerfeld, Germany)

225 was used to refine the characterisation of the particle size and obtain the particle shape of the

226 granular materials. The dispersion of the granular materials was carried out by gravity via the

227 GRADIS ${ }^{\text {TM }}$ module and the maximum resolution of the lens in the camera was $10 \mu \mathrm{m}$. A frame

228 rate of $250 \mathrm{~Hz}$ was selected in both modules for the capture of the $2 \mathrm{D}$ binary images. Three

229 shape parameters were investigated, namely: sphericity (ratio of the perimeter of a perfect circle

230 to that of the particle), aspect ratio (ratio of the minimum to maximum Feret diameters) and

231 convexity (ratio of area of the particle to its convex area). A median value of the cumulative

232 distribution, defined as the diameter of the particle that $50 \%$ of the sample mass is smaller than

$233\left(D_{50}\right)$ was used for the particle size, and for the characterisation of particle shape, the median

234 values of each of the shape parameters ( $S p_{50}$ for sphericity, $A r_{50}$ for aspect ratio and $C v_{50}$ for

235 convexity) obtained from the respective cumulative distributions were reported. It was assumed

236 that the number of images analysed in the characterisations of both particle size and shape was

237 large enough such that analysis of additional images would not change the median values.

238 
239 A scanning electron microscope (SEM, Zeiss Leo 1530 FEG, Jena, Germany) was used to 240 qualitatively investigate the microscopic and nanoscopic surface morphology of the granular 241 materials. The samples were first sputtered with a thin layer (thickness $\sim 5 \mathrm{~nm}$ ) of a Gold242 Palladium alloy in the ratio of 3:2 using the BAL-TEC SCD 005 sputter coater. Images were 243 acquired at working distances of $5-10 \mathrm{~mm}$ and at an acceleration voltage of $5 \mathrm{kV}$.

245 The characterisation of surface roughness was carried out using an optical white light 246 profilometer, Fogale Microsurf 3D, model M3D 3000 (Fogale Nanotech, Nîmes, France). A 247 Mirau interferometric objective lens with standard magnification of $50 \times$ was used to scan 25 248 randomly selected areas on the silanised and powder-coated sands with optimum lateral and 249 vertical resolutions of $0.184 \mu \mathrm{m}$ and $3 \mathrm{~nm}$ respectively. To account for the differences in particle 250 sizes of the sands, the square-shaped scanned areas were reduced in decreasing order of 251 particle sizes. The scanned areas of the $600-1180 \mu \mathrm{m}, 212-300 \mu \mathrm{m}$ and $63-212 \mu \mathrm{m}$ particle 252 sizes were respectively set as $80 \times 80 \mu \mathrm{m}, 40 \times 40 \mu \mathrm{m}$ and $35 \times 35 \mu \mathrm{m}$. The influence of 253 curvature was excluded from all measurements of surface roughness, which was evaluated 254 using the proprietary result viewer software, Fogale 3D Viewer (Version 2006-06) according to 255 the following equation:

256

$257 \quad R_{\mathrm{a}}=\frac{1}{N} \sum_{\mathrm{n}=1}^{N}\left|R_{\mathrm{n}}\right|$

259 with $R_{a}$ representing the center-line average, $N$ corresponding to the total number of pixels in 260 the scanned area and $R_{\mathrm{n}}$ representing the height of each pixel with respect to the baseline.

262 4. Results and discussion

2634.1 Influence of silanisation and powder coating on hydrophobicity

264 The CAs of the sands and silica powder before silanisation were found to be $\sim 10^{\circ}$. Figure 3 265 illustrates the relationship between concentration of DMDCS and CAs for the coarse sand, 266 medium sand, fine sand and silica powder following the silanisation reactions. Adding DMDCS 267 improves the non-wettability of the soils significantly, the contact angle increasing for all sands 

coarse sand due to the comparatively smaller surface area (dark solid line). The CA eventually

270 plateaued indicating that further increase in CA solely due to changes in surface chemistry was

271 not feasible. The critical concentration of the fine sand, medium sand and the silica powder was 272 found to be $0.53 \%$ and that of the coarse sand was $0.0795 \%$. At the relevant critical 273 concentrations, the CAs of the coarse, medium and fine sands were $109^{\circ}, 122^{\circ}$ and $127^{\circ}$

274 respectively. These granular materials were more hydrophobic than the silanised microscope 275 slides $\left(103^{\circ}\right)$ and less than the silanised silica powder at the critical concentration $\left(137^{\circ}\right)$.

277 For the powder-coated sands, both the sands and the silica powder were initially silanised at $2780.53 \%(C)$ and mixed at a 1 to 1 mass ratio. The CAs measured with the powder coated sands 279 all showed increases: the coarse, medium and fine sands had CAs of $123^{\circ}, 124^{\circ}$ and $128^{\circ}$ 280 corresponding to increases of $14^{\circ}, 2^{\circ}$ and $1^{\circ}$ respectively compared to the simply silanised sands (Figure 4). These results indicate that the enhancement of CAs as a result of the powder 282 coating procedure is dependent of the particle size of the sand, the coarser the sand, the 283 greater the increase in hydrophobicity.

284

285 The effect of increasing $C$ on the CA for a fixed mass mixing ratio of 1 to 1 was investigated 286 next. When $C$ was increased to $2 C$ and $7 C$, a general increase in $C A$ was recorded with the 287 medium and fine sands (Figure $4 \mathrm{~b}$ and $\mathrm{c}$ ). However, with the coarse sand, there was 288 comparatively no change in CA; the CAs measured at $C$ and $7 C$ being $123^{\circ}$ and $122^{\circ}$ 289 respectively (Figure 4a). The amount of silica powder was then increased by raising the mass 290 mixing ratio to 1 to 3 . Despite a similar increase in CAs being observed with this new ratio 291 compared to the simply silanised sand, increasing the mass of silica powder relative to the mass 292 of sand did not further enhance the CAs of the powder-coated sands, e.g. with the coarse sand, 293 the $C A$ at concentration $2 C$ at both mixing ratios was $120^{\circ}$.

2954.2 Influence of silanisation and powder coating on particle size, particle shape and 296 surface roughness 
297 There was no measured change in particle size between the pure and silanised sands; the $D_{50}$

298 of the sands were $768 \mu \mathrm{m}$ for the coarse sand, $247 \mu \mathrm{m}$ for the medium sand and $177 \mu \mathrm{m}$ for the

299 fine sand (Figure 5a, c and e). This indicates that the thickness of the PDMS coatings achieved

300 on the silanised sands were less than the resolution of the lens in the DIA $(10 \mu \mathrm{m})$. For the

301 powder-coated sands, the coarse sand showed an increase in $D_{50}$ as $C$ increased. An increase

302 of $88 \mu \mathrm{m}$ as the coarse sands were powder-coated was observed at a concentration of $7 C$ at

303 the 1 to 1 mixing ratio when compared to the silanised sands (Figure 5a). With the medium and

304 fine sands, the changes in $D_{50}$ were close to the resolution of the lens in the DIA and insensitive

305 to changes in $C$ (Figure $5 \mathrm{c}$ and e). For instance, the $D_{50}$ of the fine sand at the 1 to 1 mixing

306 ratio were as follows: $169 \mu \mathrm{m}$ (powder-coated at $C$ ), $177 \mu \mathrm{m}$ (powder-coated at $2 C$ ) and $182 \mu \mathrm{m}$

307 (powder-coated at 7C). An increase in fine content due to the silanised silica powder 308 aggregating after the powder coating procedure was observed with the powder-coated medium 309 and fine sands (Figure $5 \mathrm{c}$ and e), but not with the powder-coated coarse sand.

310

311 Similarly, a comparison of the median values of the shape parameters of the silanised and 312 powder-coated sands show that regardless of particle size and mixing ratio, no change in 313 particle shape was observed. Figure $5 b, d$ and $f$ illustrates the cumulative distributions of the 314 three shape parameters for the hydrophilic, silanised and powder-coated sands at a 1 to 1 315 mixing ratio.

316

317 As for the effect of silanisation and powder coating on surface roughness, qualitative analysis of 318 the sands obtained using the SEM microphotographs showed the adhered silica powders to the 319 silanised sand (Figure 6a) while the optical white light profilometer was used to report 320 quantitatively the surface roughness of the sands (Figure $6 \mathrm{~b}$ and $\mathrm{c}$ ). The $R_{\mathrm{a}}$ of the hydrophilic 321 coarse, medium and fine sands were $559 \mathrm{~nm}, 621 \mathrm{~nm}$ and $689 \mathrm{~nm}$ respectively. The silanisation 322 of the sands at a concentration of $0.53 \%$ resulted in an overall smoothening of the coarse $\left(R_{a}=\right.$ $323477 \mathrm{~nm})$, medium $\left(R_{\mathrm{a}}=416 \mathrm{~nm}\right)$ and fine $\left(R_{\mathrm{a}}=477 \mathrm{~nm}\right)$ sands due to the formation of the 324 PDMS coating on the sands. These data suggest that besides molecular interactions such as 325 Van der Waal forces occurring within the interface of the silanised sands and silanised silica 326 powder, an increase in contact area (due to the smoothening of the sands) promotes adhesion. 

surface roughness was shown to enhance adhesion. Powder coating the silanised sands resulted in an increase in $R_{a}$ with all sands. Regardless of the mixing ratio, an increase in $R_{a}$ as $C$ increased to $2 C$ and $7 C$ was recorded. Compared to the silanised coarse sand ( $\left.R_{a}=477 \mathrm{~nm}\right)$, the $R_{a}$ values of the powder-coated coarse sand at $C, 2 C$ and $7 C$ at a 1 to 1 mixing ratio were $707 \mathrm{~nm}, 747 \mathrm{~nm}$ and $840 \mathrm{~nm}$ respectively. However, when the mixing ratio switched from 1 to 1

333 to 1 to $3, R_{a}$ values became lower due to an increase in the silica powder adhered to the sands, causing the deviations of the asperities (silica powder) from the datum to diminish, thus leading to a decrease in $R_{\mathrm{a}}$ (Figure $7 \mathrm{a}$ and $\mathrm{b}$ ).

\subsection{Comparison of contact angles to theoretical models}

338 According to equation (4), the ratio of $\cos \theta_{\mathrm{w}}$ to $\cos \theta_{\mathrm{y}}$ should equal the calculated ratio of actual to projected area of the solid, $r$, for the silanised and powder-coated sands to adhere to Wenzel's model. The silanised microscope slides were considered as ideal solids and $\theta_{y}$ taken as $103^{\circ}$. Because the roughness as characterised by the the optical white light profilometer cannot be translated into the roughness factor used in Wenzel's equation, $r$ was calculated using the actual and projected areas from the 3D profiles generated with the optical white light profilometer. The effect of curvature was included in the measurements of the actual areas and $r$ was obtained by dividing the actual area with the projected area (Figure 8). The plot of cos $\theta_{w} / \cos \theta_{y}$ versus $r$ with $\theta_{w}$ equals to the experimentally measured CAs is shown in Figure 9. All

347 data points lied above the unity line showing that $\cos \theta_{w} / \cos \theta_{y}$ exceeded the $r$. These results demonstrate that because the silanisation and the powder coating procedure increases the values of $\mathrm{CA}$ and $r$, the water drops dispensed on the sands do not fully penetrate the surface protrusions. This suggests that the increases in CAs recorded with the silanised and powdercoated sands may not only be due to surface chemistry and surface roughness but also to the presence of air in between the water drops and the sands.

354 If using the Cassie-Baxter model (equation (5)) on the other hand, it is assumed that any 355 change in hydrophobicity can only be linked to the surface chemistry and air, i.e. the influence of particle size, particle shape and surface roughness are not taken into account. From equation 
357 (5), it can be deduced that increasing the area fraction of air in contact with the water drop, (1-

$358 f_{1}$ ) leads to an increase in CA. Enhancements in CAs on flat surfaces have previously been 359 attributed to $\left(1-f_{1}\right)$, for example, Yu et al. (2019) illustrated that a rise in CA of $15^{\circ}$ on 360 hydrophobic glass surfaces compared to smooth surfaces was because of $\left(1-f_{1}\right)$ increasing to 361 0.41. In our study, a similar value was obtained after the powder coating procedure with the 362 coarse sands: the $\mathrm{CA}$ of the silanised sands was $109^{\circ}$ and the resulting powder coating at 1 to 1 363 mixing ratio at $C$ led to a CA of $123^{\circ}$. This corresponds to $\left(1-f_{1}\right)$ equal to 0.13 and 0.41 for the 364 silanised and powder-coated sands respectively. This means that for this sand, air occupies an 365 additional $28 \%$ of area fraction when powder-coated.

366

367 The comparison with both theoretical models thus suggests that coating the sand particles with 368 powder results in entrapment of air on the surface of the grains, contributing to enhance the 369 hydrophobicity of the sand further than what is achieved by simple chemical treatment.

370

\section{5. Conclusion}

372 This paper presents a new methodology to optimise the hydrophobicity of granular materials by

373 powder coating sands of variable sizes. The most significant enhancement in hydrophobicity as

374 a consequence of the powder coating procedure was observed for the coarse sand, followed by

375 the medium and fine sands. Compared to the silanised sands: 1) A maximum increase of $14^{\circ}$ in 376 CAs (from $109^{\circ}$ to $123^{\circ}$ ) was recorded with the powder-coated sands. 2) There was no change 377 in particle shape recorded when the sands were powder-coated. 3) An increase in particle size 378 was observed only with the coarse sand whereas the medium and fine sands showed only 379 increases in the fines content. 4) The surface roughness of all sands increased. The concept of 380 physically modifying granular materials to tune hydrophobicity can potentially be extended to a 381 wide variety powders of different shapes and of finer sizes (e.g. nanosilica) for their eventual 382 deployment in ground engineering. 


\section{Acknowledgements}

385 This work was supported by the General Research Fund (Grant 17203417) from the Research 386 Grants Council of Hong Kong Special Administrative Region, China. 
Bachmann, J., Ellies, A. and Hartge, K. (2000) Development and application of a new sessile drop contact angle method to assess soil water repellency. J. Hydrol. 231-232: 66-75.

Bachmann, J., Woche, S. K., Goebel, M. O., Kirkham, M. B. and Horton, R. (2003) Extended methodology for determining wetting properties of porous media. Water resources research 39(12).

393

Bachmann, J. and McHale, G. (2009) Superhydrophobic surfaces: a model approach to predict contact angle and surface energy of soil particles. Eur. J. Soil Sci. 60(3): 420-430.

Bardet, J., Jesmani, M., Jabbari, N. and Lourenco, S. D. N. (2014) Permeability and 396 compressibility of wax-coated sands. Geotechnique 64(9): 752-755.

Bentz, D. P., Ferraris, C. F., Jones, S. Z., Lootens, D. and Zunino, F. (2017) Limestone and silica powder replacements for cement: Early-age performance. Cem. Concr. Compos. 78: 43-56.

400

Blossey, R. (2003) Self-cleaning surfaces—virtual realities. Nat. Mater. 2(5): 301.

401 Brown, H.R. (2000) Polymer adhesion. Mater. Forum. 24: 49-58.

402 Camino, G., Lomakin, S. M. and Lazzari, M. (2001) Polydimethylsiloxane thermal degradation 403 Part 1. Kinetic aspects. Polymer 42(6): 2395-2402.

404 Carrillo, M. L. K., Yates, S. R. and Letey, J. (1999) Measurement of initial soil-water contact 405 angle of water repellent soils. Soil Sci. Soc. Am. J. 63(3): 433-436.

406 Cassie, A. B. D. and Baxter, S. (1944) Wettability of porous surfaces. Trans. Faraday Soc. 40: $407 \quad 546-551$

408 Chan, C. S. H. and Lourenço, S. D. N. (2016) Comparison of three silane compounds to impart 409 water repellency in an industrial sand. Geotechnique Lett. 6(4): 263-266.

410 Darmanin, T. and Guittard, F. (2015) Superhydrophobic and superoleophobic properties in 411 nature. Mater. Today 18(5): 273-285.

412 Dekker, L. W. and Ritsema, C. J. (1994) How water moves in a water repellent sandy soil: 1. 413 Potential and actual water repellency. Water Resour. Res. 30(9): 2507-2517.

414 Doerr, S. H., Shakesby, R. A. and Walsh, R. P. D. (2000) Soil water repellency: its causes, 415 characteristics and hydro-geomorphological significance. Earth-Sci. Rev. 51(1-4): 33-65. 
416 Dong, Y. and Pamukcu, S. (2015) Thermal and electrical conduction in unsaturated sand 417 controlled by surface wettability. Acta Geotechnica 10(6): 821-829.

418 Feng, L., Li, S., Li, Y., Li, H., Zhang, L., Zhai, J., Song, Y., Liu, B., Jiang, L. and Zhu, D. (2002) 419 Super-hydrophobic surfaces: from natural to artificial. Adv. Mater. 14(24): 1857-1860.

420 Fuller, K. N. G. and Tabor, D. (1975) The effect of surface roughness on the adhesion of elastic 421 solids. Proceedings of the Royal Society A: Mathematical, Physical and Engineering Sciences. 345(1642): 327-342.

424

Goebel, M.-O., Woche, S. K., Bachmann, J., Lamparter, A. and Fischer, W. R. (2007) Significance of wettability-induced changes in microscopic water distribution for soil organic matter decomposition. Soil Sci. Soc. Am. J. 71(5): 1593-1599.

Grunze, M. (1999) Driven liquids. Science 283(5398): 41-42.

428

Hernandez, J., Vargas, S., Estévez, M., Vázquez, G., Zepeda, A. and Rodríguez, R. (2005) Hydrophobic modification of an expansive soil using polymers and organic compounds: a comparative study with lime. Geotechnique 55(8): 613-616.

Keatts, M.I., Daniels, J.L., Langley, W.G., Pando, M.A. and Ogunro, V.O. (2018) Apparent Contact Angle and Water Entry Head Measurements for Organo-Silane Modified Sand and Coal Fly Ash. J. Geotech. Geoenviron. Eng. 144(6): 04018030.

Kim, D., Yang, H., Kim, K. and Yun, T. (2015) Experimental investigation of evaporation and drainage in wettable and water-repellent sands. Sustainability, 7(5): 5648-5663.

Kinloch, A. J. (1980) The science of adhesion. J. Mater. Sci. 15(9): 2141-2166.

Lee, C., Yang, H. J., Yun, T. S., Choi, Y. and Yang, S. (2015) Water-entry pressure and friction angle in an artificially synthesized water-repellent silty soil. Vadose Zone J. 14(4).

Liu, D., Lourenço, S.D.N and Yang, J. (2019) Critical state of polymer-coated sands. Geotechnique 1-6.

Ng, S. H. Y. and Lourenco, S. D. N. (2016) Conditions to induce water repellency in soils with

443 Orozco, L.F. and Caicedo, B. (2017) Water migration in unsaturated partially hydrophobic soils. 444 Geotechnique Lett. 7(1): 18-23. 
445 Saulick, Y., Lourenço, S. D. N. and Baudet, B. A. (2017) A semi-automated technique for 446 repeatable and reproducible contact angle measurements in granular materials using the 447 sessile drop method. Soil Sci. Soc. Am. J. 81(2): 241-249.

448 Saulick, Y., Lourenço, S. D. N., Baudet, B. A., Woche, S. K. and Bachmann, J. (2018) Physical 449 properties controlling water repellency in synthesized granular solids. Eur. J. Soil Sci. 69(4): $450 \quad 698-709$.

451 Shirtcliffe, N. J., McHale, G., Newton, M. I., Chabrol, G. and Perry, C. C. (2004) Dual-scale 452 roughness produces unusually water-repellent surfaces. Adv. Mater. 16(21): 1929-1932.

453 Subedi, S., Kawamoto, K., Jayarathna, L., Vithanage, M., Moldrup, P., Wollesen De Jonge, L. 454 and Komatsu, T. (2012) Characterizing time-dependent contact angles for sands 455 hydrophobized with oleic and stearic acids. Vadose Zone J.11(1).

456 Tripp, C. and Hair, M. (1991) Reaction of chloromethylsilanes with silica: a low-frequency 457 infrared study. Langmuir 7(5): 923-927.

458 Wang, Z., Koratkar, N., Ci, L. and Ajayan, P. (2007) Combined micro-/nanoscale surface 459 roughness for enhanced hydrophobic stability in carbon nanotube arrays. Appl. Phys. Lett. 460 90(14): 143117.

461 Wenzel, R. N. (1936) Resistance of solid surfaces to wetting by water. Ind. Eng. Chem. 28(8): 462 988-994.

463 Yang, H., Baudet, B. A. and Yao, T. (2016) Characterization of the surface roughness of sand 464 particles using an advanced fractal approach. Proceedings of the Royal Society A: 465 Mathematical, Physical and Engineering Sciences 472(2194).

466 Young, T. (1805) An essay on the cohesion of fluids. Philos. Trans. R. Soc. London 95: 65-87.

467 Yu, L., Kang, Y., Tang, H. and Zhou, J. (2019) Functionalization of Commercial Sand Core 468 Funnels as Hydrophobic Materials with Novel Physicochemical Properties. ACS Appl. Mater. 469 Interfaces. 11(7): 7510-7521.

470 Zhang, P., Wang, S., Wang, S. and Jiang, L. (2015) Superwetting surfaces under different 471 media: Effects of surface topography on wettability. Small 11(16): 1939-1946.

472 Zhang, H. Y., Zhu, S. B., Li, M. and Zhang, X. C. (2016) Water repellency of monument soil 473 treated by tung oil. Geotech Geol Eng. 34(1): 205-216. 
474 Zimmermann, J., Reifler, F. A., Fortunato, G., Gerhardt, L. C. and Seeger, S. (2008) A simple, 475 one-step approach to durable and robust superhydrophobic textiles. Adv. Funct. Mater. 476 18(22): 3662-3669.

477 
480 Figure 1. Schematic representation of a drop of water (a) on an ideal flat solid; (b) in the Wenzel 481 state and (c) in the Cassie-Baxter state

482 Figure 2. Schematic representation of the optimisation of hydrophobicity of sands

483 Figure 3. Relationship between concentration of dimethyldichlorosilane and contact angles for 484 the coarse sand, medium sand, fine sand and silica powder

485 Figure 4. Comparison of contact angles of powder-coated sands to silanised sands for different 486 mass mixing ratios: (a) coarse, (b) medium and (c) fine sands. $C, 2 C$ and $7 C$ refer to the 487 concentrations at which the sands were initially chemically modified before powder coating. 488 Inset photographs show $10 \mu \mathrm{L}$ water drops on the silanised sands compared to the powder489 coated sands in at a mixing ratio of 1 to 1

490 Figure 5. Characterisation using dynamic image analyser: particle size distributions of (a) 491 coarse, (c) medium, (e) fine sands and particle shape distributions of (b) coarse, (d) medium, (f) 492 fine sands at a mixing ratio of 1 to 1

493 Figure 6. (a) SEM microphotographs showing the silanised and powder-coated coarse sands at 494 similar magnifications; (b) optical white light profilometry images of the silanised (left) and 495 powder-coated (right) coarse sands; (c) 2D profiles extracted from the optical white light 496 profilometry images

497 Figure 7. Change in surface roughness as the sands are powder-coated in a mixing ratio of (a) 4981 to 1 and (b) 1 to $3 . C, 2 C$ and $7 C$ refer to the concentrations at which the sands were initially 499 chemically modified before powder coating

500 Figure 8. (a) Projected and (b) actual areas of a powder-coated sand

501 Figure 9. Comparison of the ratio of contact angles to the roughness factor, $r$ for the (a) coarse

502 (b) medium and (c) fine sands. The values of $\theta_{\mathrm{w}}$ and $\theta_{\mathrm{y}}$ are the experimentally measured contact 503 angles on the sands (silanised and powder-coated) and the silanised microscope slides 504 respectively

505

506 


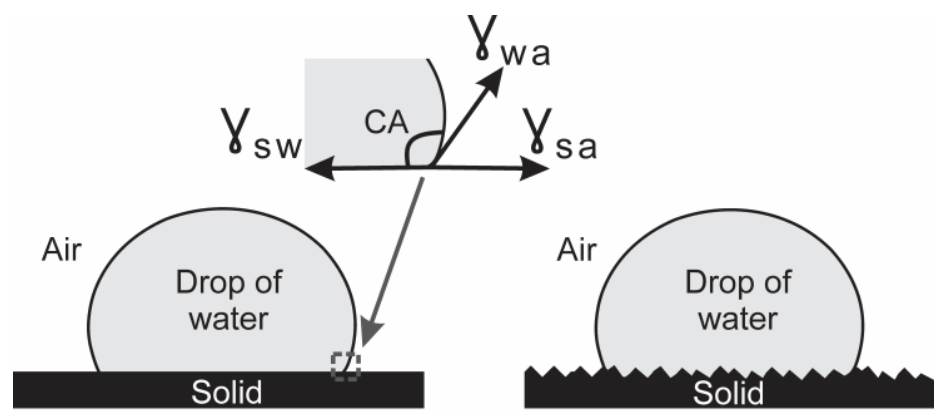

(a)

(b)

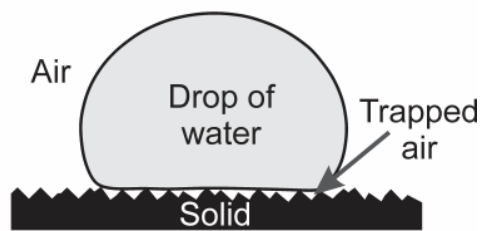

(c)

518

519 Figure 1. Schematic representation of a drop of water (a) on an ideal flat solid; (b) in the Wenzel 520 state and (c) in the Cassie-Baxter state

521

522

523

524

525

526

527

528

529

530

531

532

533

534

535

536

537

538

539

540

541

542

543

544

545

546

547

548

549

550 


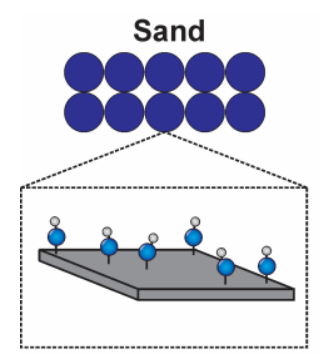

Hydrophilic sand

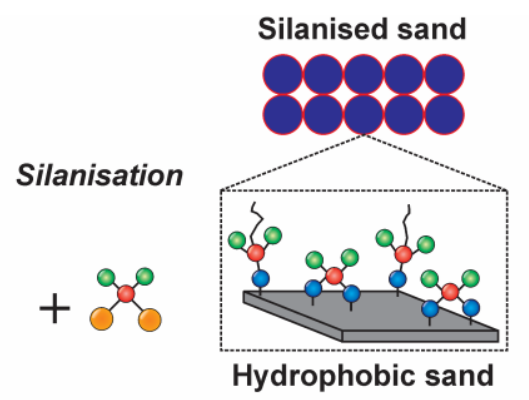

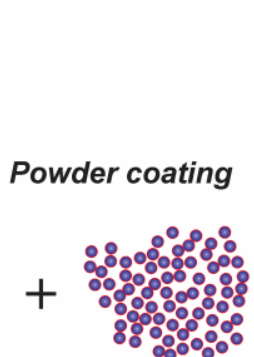
sand

Hydroxyl group $\bigcirc$ Methyl group $\bigcirc$ Silicon atom

Chlorine atom %:요 Silanised silica powder

Figure 2. Schematic representation of the optimisation of hydrophobicity of sands

553

554

555

556

557

558

559

560

561

562

563

564

565

566

567

568

569

570

571

572

573

574

575

576

577

578

579

580

581

582

583 


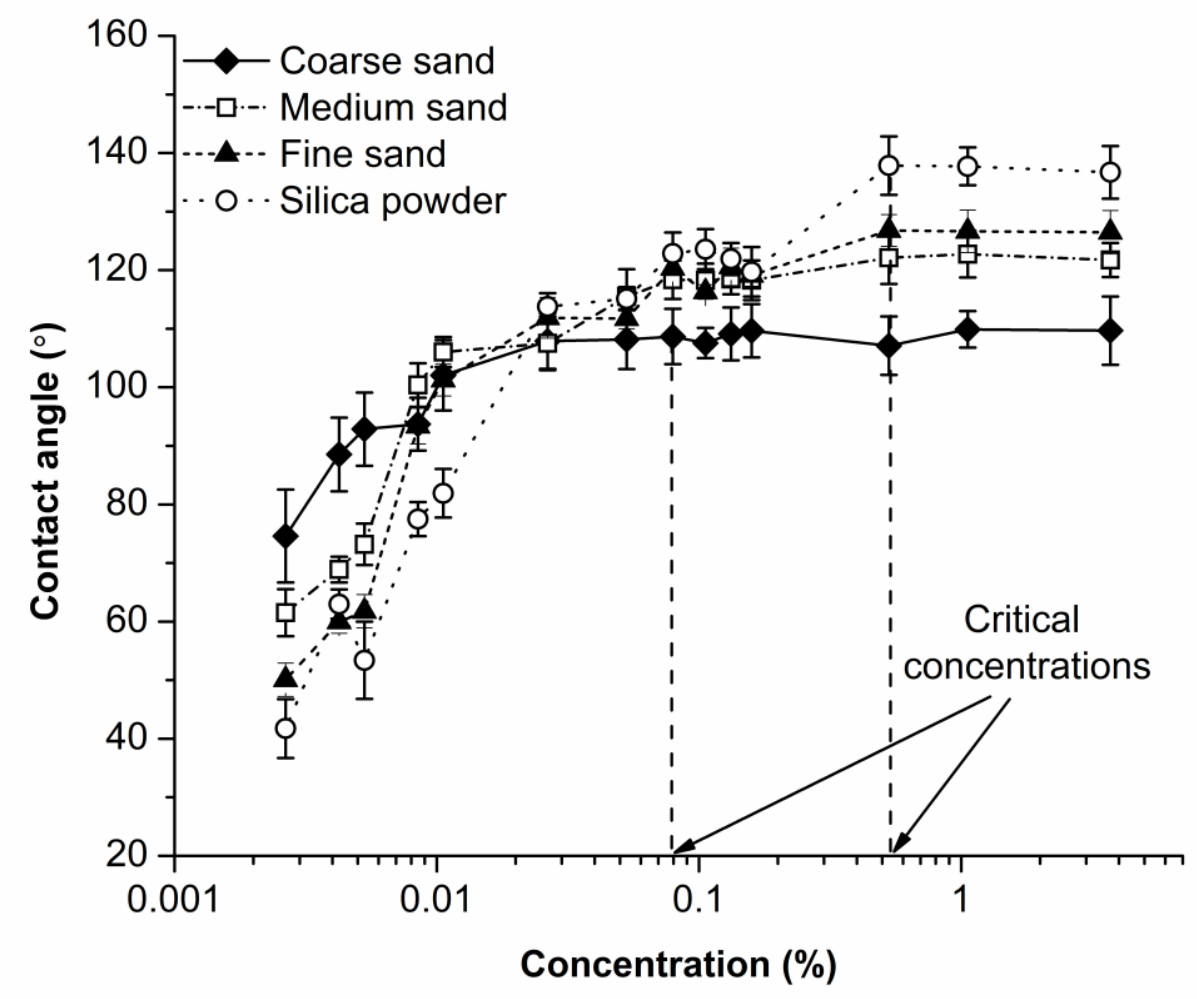

584

585 Figure 3. Relationship between concentration of dimethyldichlorosilane and contact angles for 586 the coarse sand, medium sand, fine sand and silica powder 

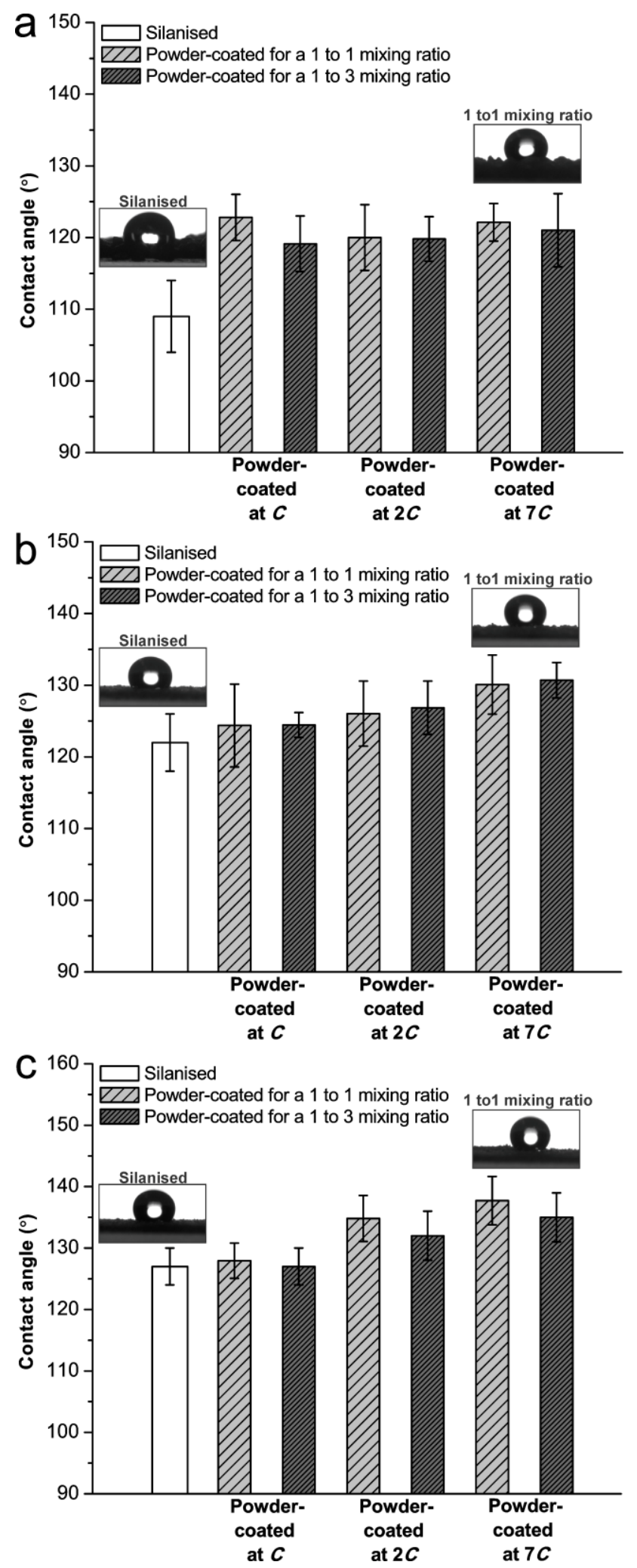

Figure 4. Comparison of contact angles of powder-coated sands to silanised sands for different 607 mass mixing ratios: (a) coarse, (b) medium and (c) fine sands. $C, 2 C$ and $7 C$ refer to the 608 concentrations at which the sands were initially chemically modified before powder coating. 609 Inset photographs show $10 \mu \mathrm{L}$ water drops on the silanised sands compared to the powder610 coated sands in at a mixing ratio of 1 to 1 

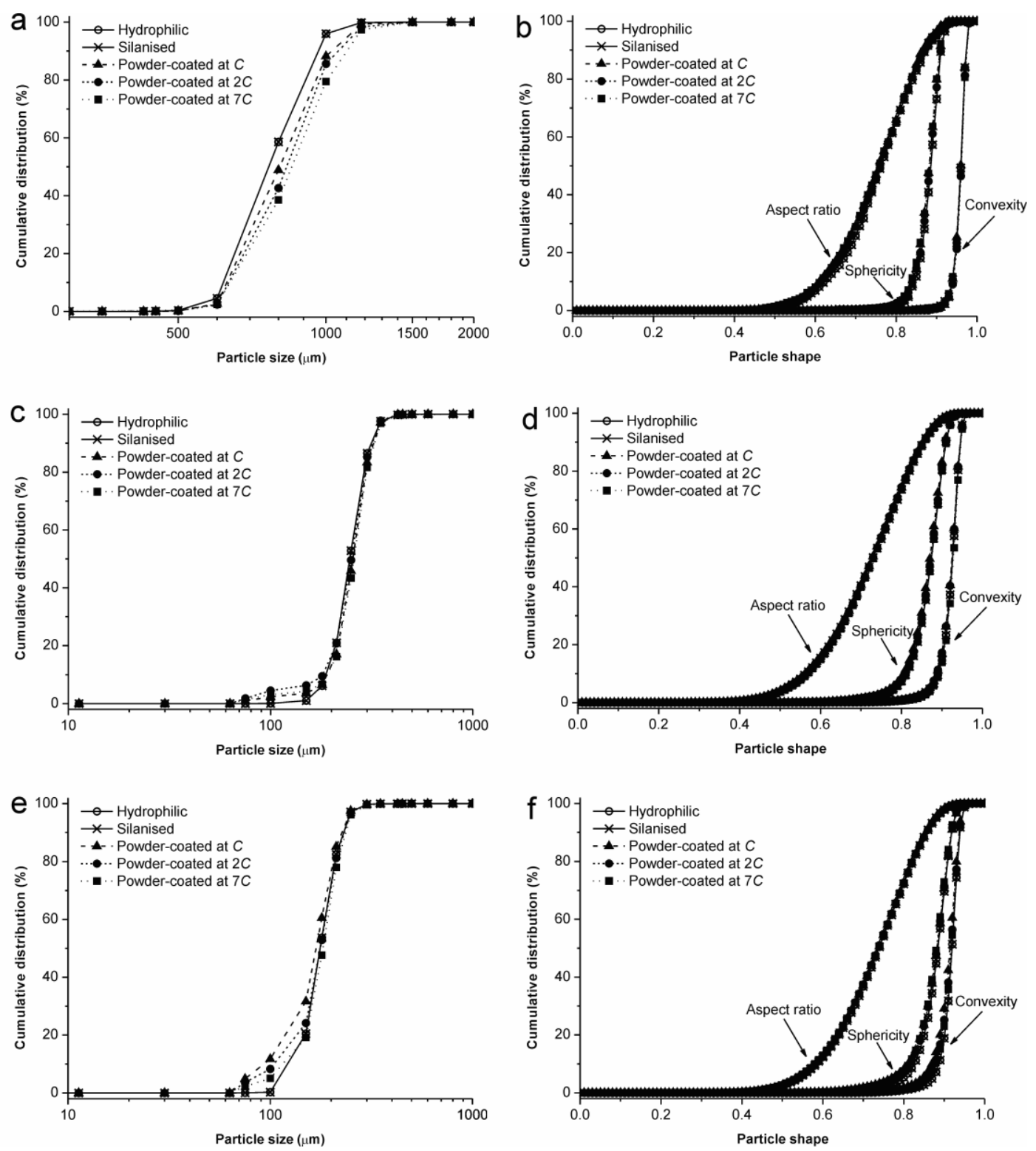

612 Figure 5. Characterisation using dynamic image analyser: particle size distributions of (a) 613 coarse, (c) medium, (e) fine sands and particle shape distributions of (b) coarse, (d) medium, (f) 614 fine sands at a mixing ratio of 1 to 1 


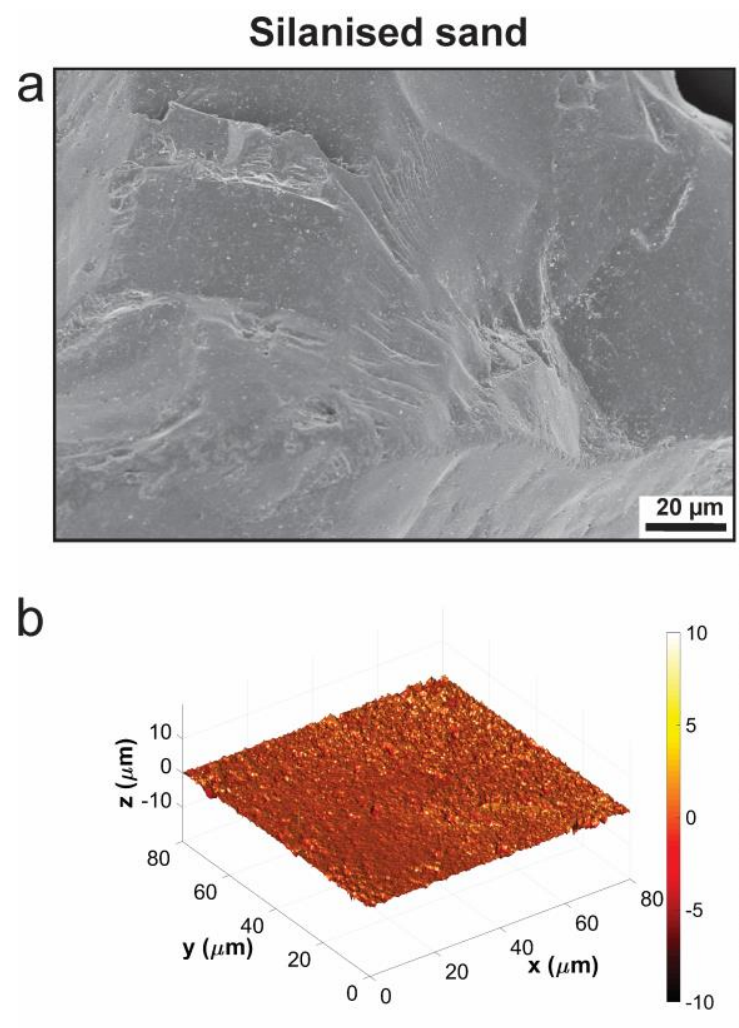

C
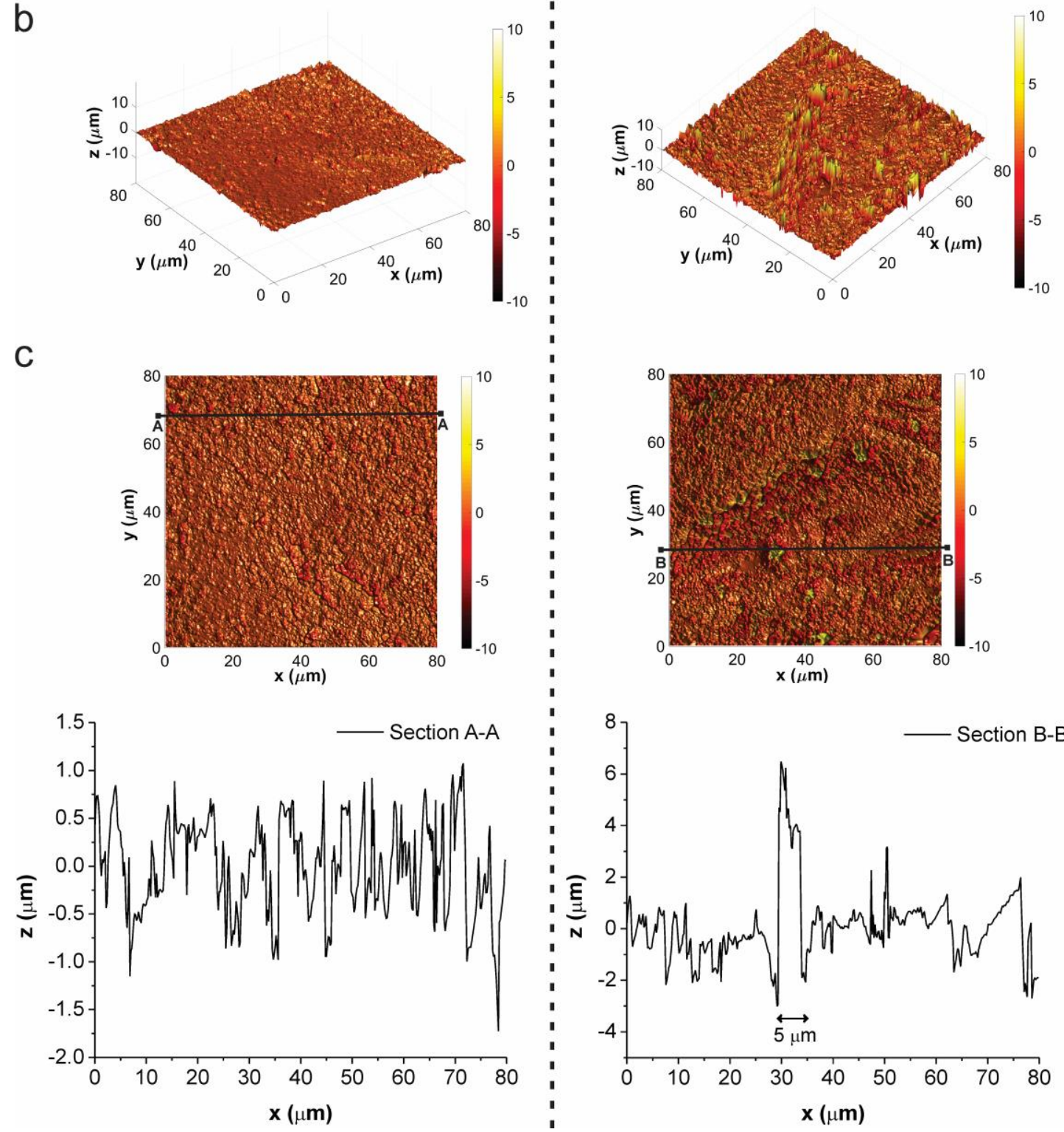

Figure 6. (a) SEM microphotographs showing the silanised and powder-coated coarse sands at

628 similar magnifications; (b) optical white light profilometry images of the silanised (left) and 629 powder-coated (right) coarse sands; (c) 2D profiles extracted from the optical white light 630 profilometry images 

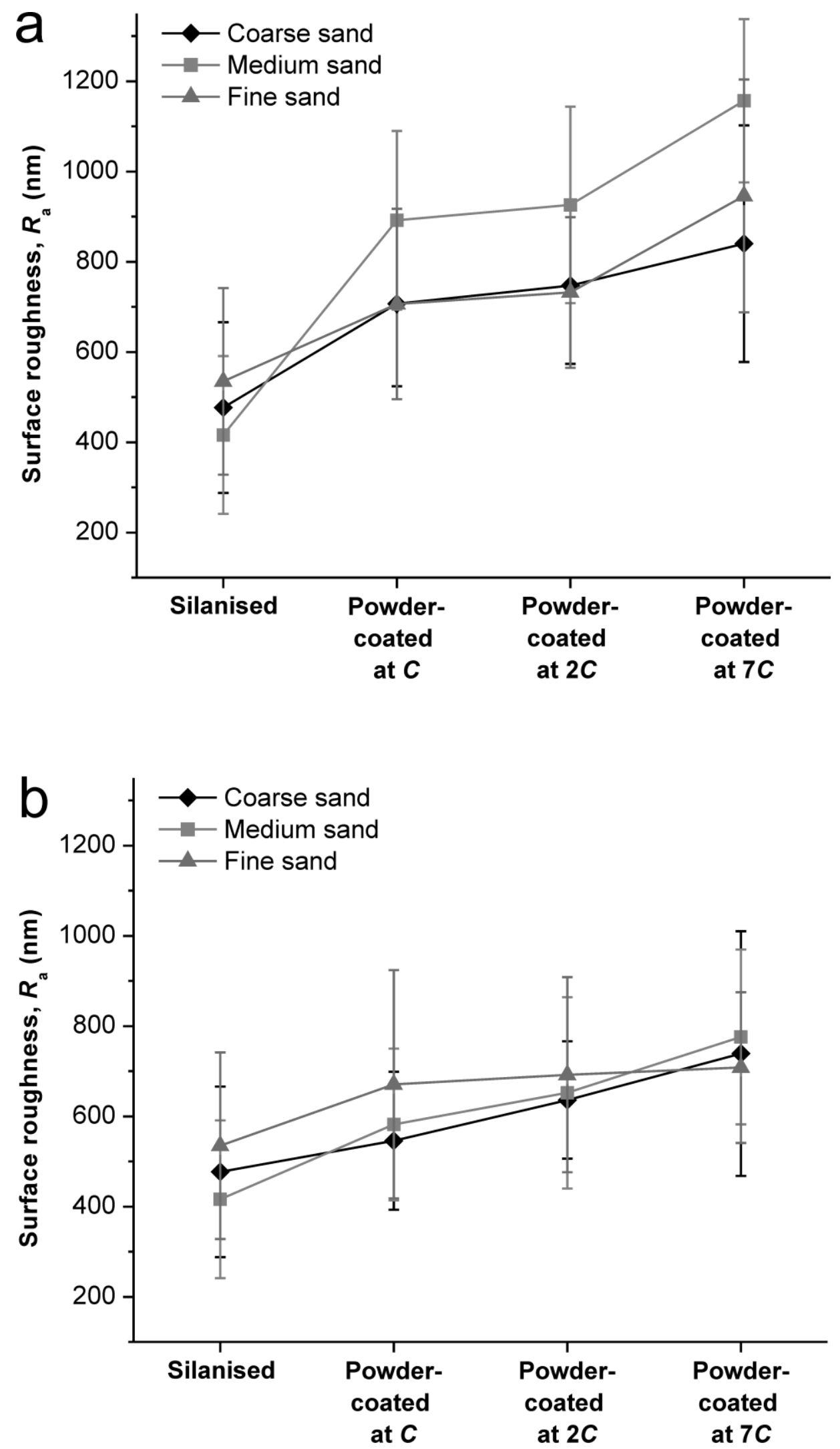

632 Figure 7. Change in surface roughness as the sands are powder-coated in a mixing ratio of (a) 6331 to 1 and (b) 1 to $3 . C, 2 C$ and $7 C$ refer to the concentrations at which the sands were initially 634 chemically modified before powder coating 

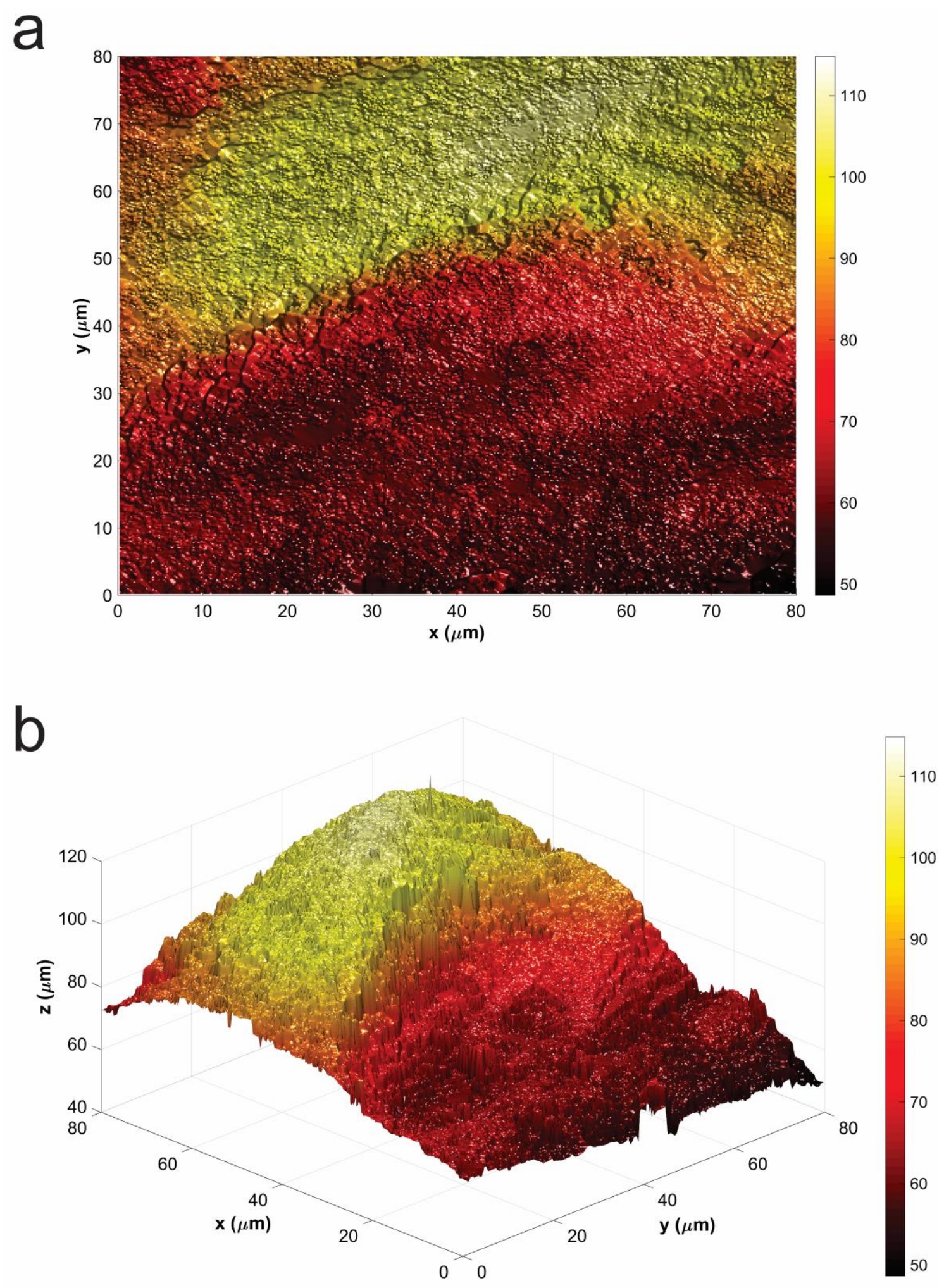

637 Figure 8. (a) Projected and (b) actual areas of a powder-coated sand 

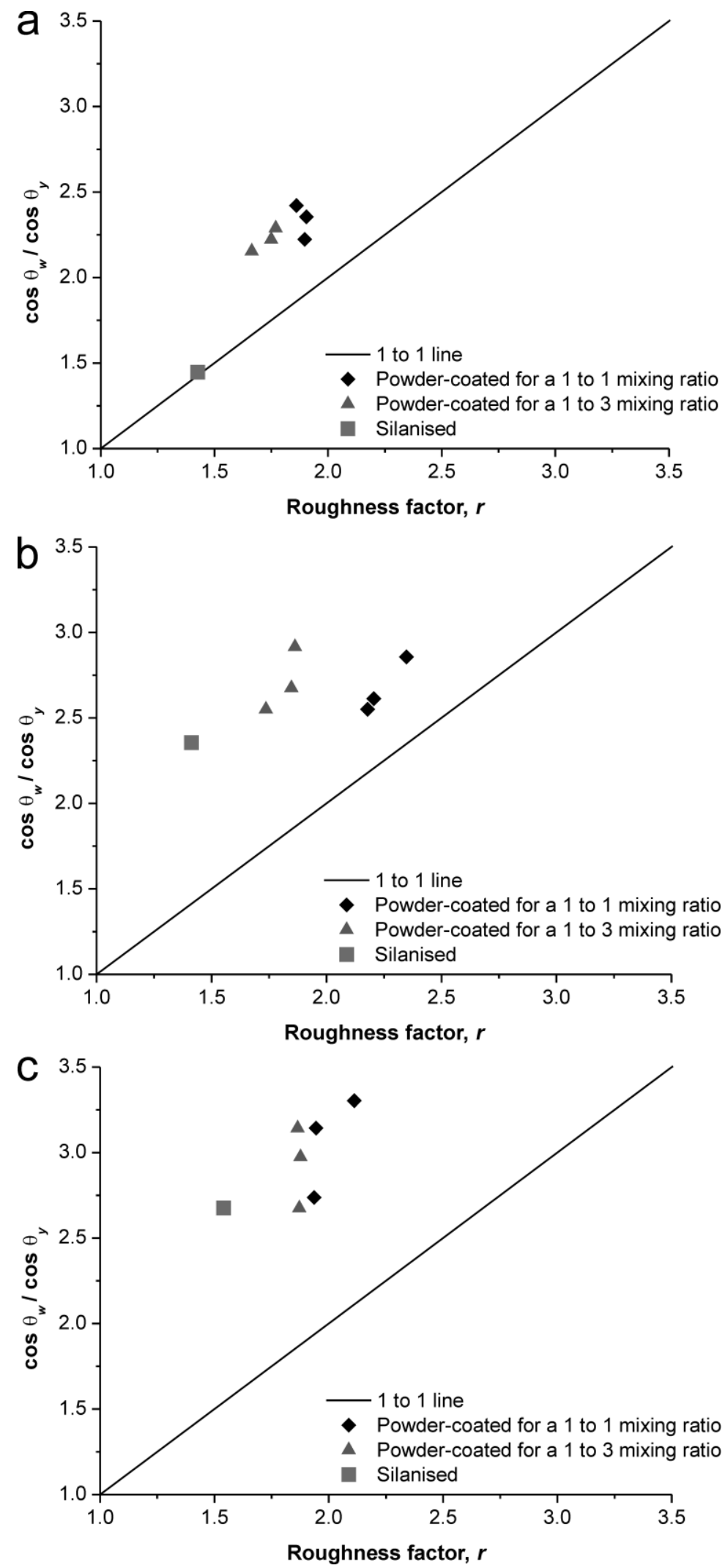
Roughness factor,

642 Figure 9. Comparison of the ratio of contact angles to the roughness factor, $r$ for the (a) coarse

643 (b) medium and (c) fine sands. The values of $\theta_{w}$ and $\theta_{y}$ are the experimentally measured contact 644 angles on the sands (silanised and powder-coated) and the silanised microscope slides 645 respectively 